\title{
Model-Based Control Strategy for Autonomous Vehicle Path Tracking Task
}

\author{
Ahmad REDA ${ }^{1}$, József VÁSÁRHELYI ${ }^{2}$ \\ ${ }^{1}$ Institute of Automation and Info-communication, Faculty of Mechanical engineering and \\ Informatics, University of Miskolc, Miskolc, e-mail: autreda@uni-iskolc.hu \\ ${ }^{2}$ Institute of Automation and Info-communication, Faculty of Mechanical engineering and \\ Informatics, University of Miskolc, Miskolc, e-mail: autreda@uni-iskolc.hu
}

Manuscript received December 2, 2020; revised December 15, 2020

\begin{abstract}
Despite the advanced technologies used in recent years, the lack of robust systems still exists. The automated steering system is a critical and complex task in the domain of the autonomous vehicle's applications. This paper is a part of project that deals with model-based control strategy as one of the most common control strategies. The main objective is to present the implementations of Model Predictive Control (MPC) for an autonomous vehicle steering system in regards to trajectory tracking application. The obtained results are analysed and the efficiency of the use of MPC controller were discussed based on its behaviour and performance.
\end{abstract}

Keywords: Model based control, Model Predictive Control (MPC), trajectory tracking, degree of freedom (DOF), dynamic vehicle model.

\section{Introduction}

In recent years with the significant increase in the number of vehicles on the roads, many types of research and reports have raised the attention to the fact of increase the risk of road safety issues such as traffic congestion and car accidents which are caused by human errors. In this context, the autonomous vehicles have gained more attention as a solution to overcome these challenges, in addition to the other benefits it provides in terms of better fuel economy provision and reducing the pollution [1], [2]. The autonomous vehicle's applications can be divided into 3 main modules: Sensing and Perception, Planning and Control. Sensing and Perception is the fundamental module that provides the information of the driving environment to the vehicle letting the system know the surrounding environment in real-time such as surrounding obstacles, velocity, and vehicle location. In the planning module, the system 
uses data provided by the previous sub-system (Sensing and Perception) to determine the safest and the most appropriate trajectory to be followed by the vehicle. In the Control module, the control strategies are applied to control the actuators of the different parts of the system in order to drive and steer the vehicle along the desired path [3]. Improving the automated steering system in order to develop a highly secure, stable and easy to drive autonomous vehicle has gained significant interest [4]. The vehicle models that can be used in trajectory tracking problems can be divided into three mains categories: Geometric, Kinematic and Dynamic vehicle models with several control strategies [5]. The paper presents preliminary research results of the proposed MPC controller based on the dynamic model and it is structured as follows: in the first section, the autonomous vehicle system review is presented. Second section describes the dynamic vehicle model and control strategy. The design of the vehicle model and the MPC controller are described in section three. In section four, the implementations and the obtained results are discussed. The conclusions of the paper are presented in section five.

\section{Autonomous vehicles model and control strategy}

The vehicle model plays an essential role in regards to the system simulations process, which is a crucial stage in the development process of the controllers. In the simulation process, the properties of the controller are defined and the parameters are tuned in order to achieve the best performance. The vehicle (like robot) model can be divided into two main classes, holonomic system and non-holonomic system based on the number of controllable degrees of freedom (DOF) against the total number of DOF. In a holonomic system, the number of controllable degrees of freedom is equal to the total number of DOF. In contrast, a system with number of controllable degrees of freedom less than the total number of DOF is called a non-holonomic system. It is worth to mention that most of the vehicles are considered as non-holonomic due to the fact that only two degrees of freedom are controllable (the lateral positions and the longitudinal directions) [6], [7]. One more thing to take into consideration is that the vehicle model can be reduced to be two - tire models which is called a bicycle model. In bicycle models the right and the left tires are assumed to have the same behaviour. Trajectory tracking tasks based on [8], [9], can be divided into three main motion tasks which are, trajectory tracking, path following and point to point motion. 


\subsection{Dynamic Vehicle Model}

Kinematic, Geometric and Dynamic models are the three main types of path tracking tasks. Unlike Geometric and kinematic model, the dynamic model considers the internal and external forces and describes the motion of the vehicle with respect to its position, velocity and acceleration. Fig. 1 shows the dynamic vehicle model, where $\theta$ is the vehicle heading, $v$ is vehicle velocity, $\delta_{R}$ and $\delta_{F}$ denote the rear and front steering angle respectively, $F_{F}$ and $F_{R}$ are the lateral forces that are applied on the front and rear tires respectively, $L$ is the wheelbase of the vehicle, $L_{F}$ and $L_{R}$ are the rear and front half-wheelbases respectively, $G$ is the gravity force, $\beta_{F}$ and $\beta_{R}$ are the sideslip angles of the front and rear wheels respectively [10], [11].

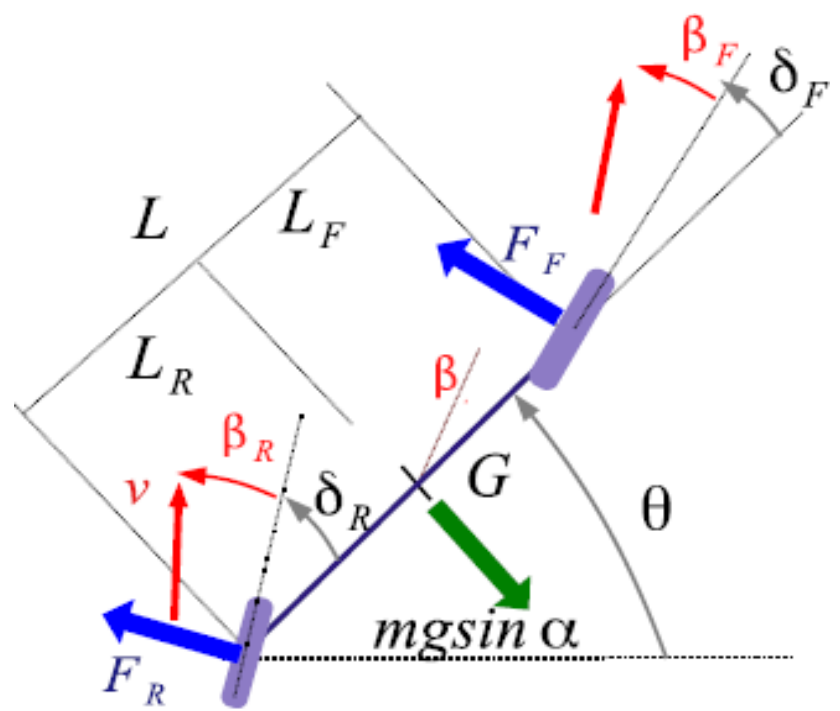

Figure 1: Dynamic vehicle model

\subsection{Dynamic Controller}

In the path tracking task, the control law of the dynamic controller includes the dynamic properties of the system. Taking the effects of the vehicle dynamics into consideration naturally makes the dynamic controllers more efficient and stable compared to geometric and kinematic controllers [12]. However, dynamic feedback (such as the torque) is required for this type of control strategy, which in turn requires a special type of sensors and more data processing. Several studies can be found regarding the use of the dynamic control strategy for autonomous vehicle tasks [13], [14]. In this paper, Model 
Predictive Control strategy was used to control the autonomous vehicle model as a dynamic control strategy. Model Predictive Controller (MPC) is an optimization strategy for model-based control, and it can be implemented for linear and nonlinear systems. The optimization process is achieved by selecting the optimal values of the plant model which in turn provides the optimal performance in terms of tracking the reference output. MPC controller uses the plant model to simulate the vehicle path (for vehicle path tracking task) in the next $\mathrm{P}$ time steps (prediction horizon), meaning that it looks forward to make the predictions. MPC strategy uses different scenarios to achieve the optimization, where the optimal scenario is the one that minimizes the error between the predicted and the reference trajectory. MPC is an effective strategy for the systems where the model dynamics are unchanged [15].

\section{Design of the MPC controller}

Model Predictive Controller is used to control the front steering angle of the vehicle by means of adjusting and driving the lateral deviation $(d)$ and the relative yaw angle $(\theta)$ to be very close to zero (see Fig. 2). To design an MPC controller, the model of the vehicle is created in the first step, and the parameters of the MPC are designed in the second step.

\subsection{Design of the Dynamic Model of the Vehicle}

In this paper, the bicycle model is used to describe the dynamics of the vehicle. Fig. 2 shows the global position of the vehicle in $(\mathrm{X}, \mathrm{Y})$ axes. $v_{x}$ is the longitudinal velocity and $v_{y}$ is the lateral velocity. The vehicle model is described with the following parameters: total mass of the vehicle $(m)$, yaw moment of the vehicle inertia $\left(l_{z}\right)$, the distance from the front tires to the centre of gravity $\left(l_{f}\right)$, the distance from the rear tires to the centre of gravity $\left(l_{r}\right)$, the cornering stiffness of front tire $\left(c_{f}\right)$, and the cornering stiffness of rear tire $\left(c_{r}\right)$. The lateral motion and yaw motion are determined by the fundamental laws of motion, meaning that they are determined by forces which are (in this case) the forces that are applied on front and rear tires [16], [17]. 


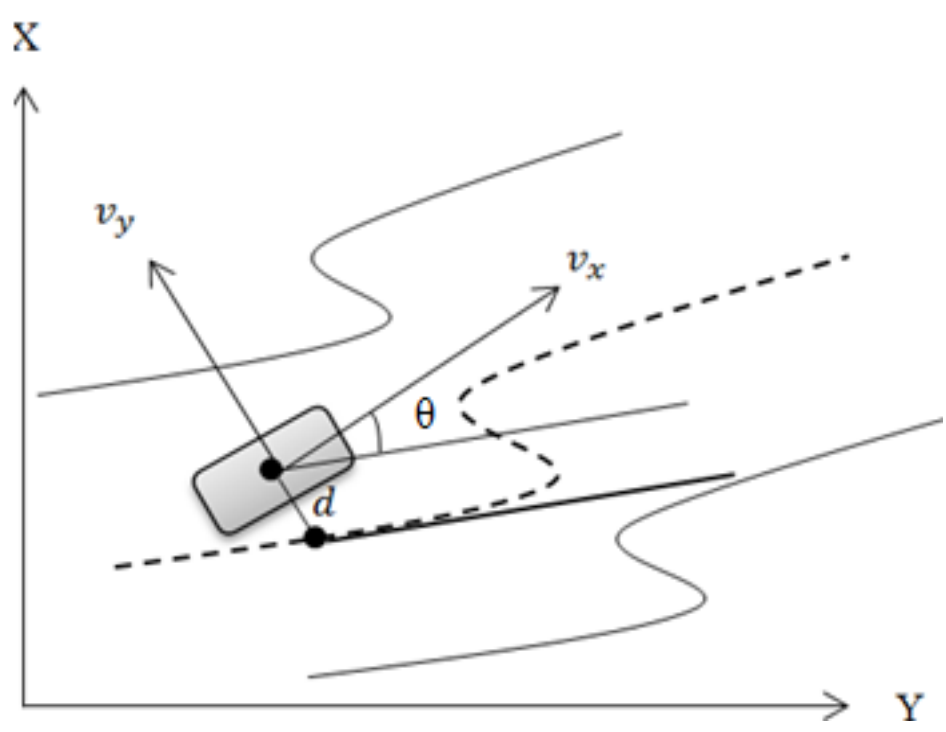

Figure 2: Position of vehicle model

Equations (1-a), (1-b) and (1- c) describe the lateral dynamics of the vehicle, where $m$ is the mass of the vehicle, $a_{y}$ is the acceleration in lateral direction y, $\omega$ is the yaw rate, $F_{F}$ and $F_{R}$ are the lateral forces that are applied on the front and rear tires, respectively.

$$
\begin{gathered}
m a_{y}=\sum_{i} F_{y, i}=F_{F}+F_{R} \\
a_{y}=\dot{v}_{y}+\omega v_{x} \\
m\left(\dot{v}_{y}+\omega v_{x}\right)=F_{F}+F_{R}
\end{gathered}
$$

The yaw dynamics of the vehicle is described by the sum of the all moments about the central gravity G (moments generated by front and rear tires) as (2) shows. Regarding to linear tire model, the tire forces are proportional to the corresponding slip angles $\left(\beta_{F}, \beta_{R}\right)$ and the corner stiffness of the front and rear wheels $\left(c_{f}, c_{r}\right)$ as equations (3-a) and (3-b) describe.

$$
\begin{gathered}
l_{z} \dot{\omega}=l_{f} F_{F}+l_{r} F_{R} \\
F_{F}=c_{f} \beta_{F} \\
F_{R}=c_{r} \beta_{R}
\end{gathered}
$$

The bicycle model can be described as continuous state space representation as it is shown in (4) where: 
States: $x_{1}=v_{y}, x_{2}=\theta, x_{3}=\omega$.

Input: $\mathrm{u}=\delta$.

$$
\frac{d}{d t}\left[\begin{array}{l}
x_{1} \\
x_{2} \\
x_{3}
\end{array}\right]=\left[\begin{array}{c}
\dot{y} \\
\dot{\theta} \\
\dot{\omega}
\end{array}\right]\left[\begin{array}{ccc}
\frac{-\left(2 C_{f}+2 C_{r}\right)}{m v_{x}} & 0 & -v_{x}-\frac{-\left(2 c_{f} l_{f}-2 c_{r} l_{r}\right)}{m v_{x}} \\
0 & 0 & 1 \\
\frac{-\left(2 C_{f} l_{f}-2 C_{r} l_{r}\right)}{I_{z} v_{x}} & 0 & \frac{-\left(2 C_{f} l_{f}+2 C_{r} l_{r}{ }^{2}\right)}{I_{z} v_{x}}
\end{array}\right]\left[\begin{array}{c}
x_{1} \\
x_{2} \\
x_{3}
\end{array}\right]+\left[\begin{array}{c}
\frac{2 c_{f}}{m} \\
0 \\
\frac{2 c_{f} l_{f}}{I_{z}}
\end{array}\right] \delta(4)
$$

The full discretized model for MPC controller is obtained by discretizing the continuous state space model using sample time $\left(T_{s}=0.1\right)$. In the next step the parameters of the controller need to be determined.

\subsection{Design of the MPC model}

The first step in the process of designing the MPC controller is to set the channels and the signal types of the plant model. In other words, to set the input and output channels. The signal types of input channels are: Manipulated variables (MV), Measured Disturbances (MD) and Unmeasured Disturbances (UD), whereas output channels are: Measured Outputs (MO) and Unmeasured Outputs (UO). In default configuration, all the inputs are considered as manipulated variables and all outputs are measured outputs. The MPC plant model in this paper was designed with two inputs, therefore the first and second input channels were set as MV for steering angle and MD for road yaw angle rate. respectively. In addition to the inputs, the model has four measured outputs, which are: lateral deviation, relative yaw angle, lateral velocity, and yaw rate $(\omega=\dot{\psi})$ as it is shown in Fig. 3. Yaw rate is the rotation rate of the car or in other words the changing rate of the heading angle of the car. Generally speaking, in the course of designing the parameters of an MPC controller, several recommendations can be taken into consideration [15]. In addition to design the parameters, the constraints of the manipulated variable (steering angle) were set as Table 1 shows. 


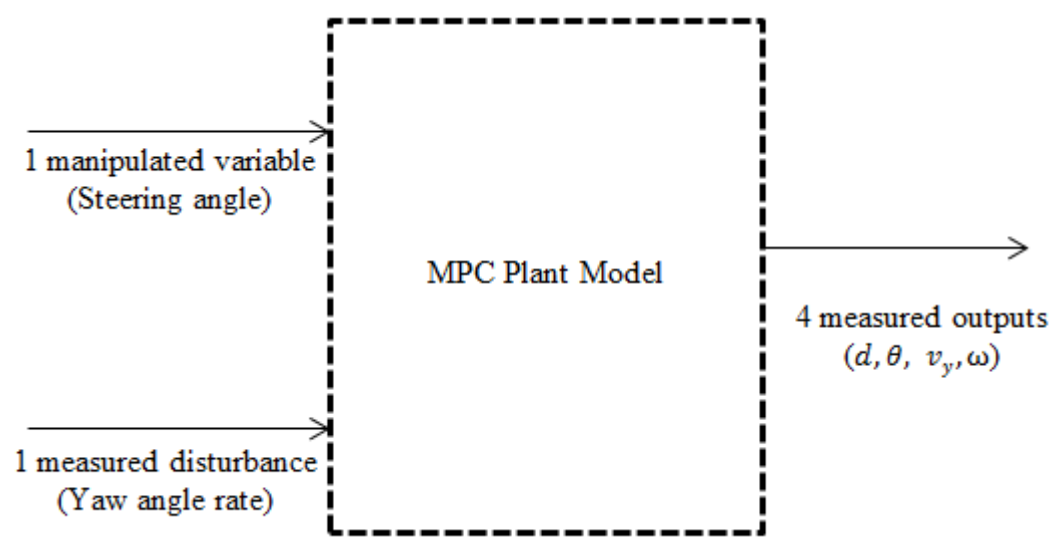

Figure 3: Inputs and outputs of MPC Plant model.

Table 1: Constraints and Parameters of the MPC controller

\begin{tabular}{|c|c|}
\hline \multicolumn{2}{|c|}{ Parameters } \\
\hline Sample Time $\left(T_{s}\right)$ & 0.1 seconds \\
\hline Prediction Horizon $(\mathrm{P})$ & 20 seconds \\
\hline Control Horizon $(\mathrm{M})$ & 20 seconds \\
\hline \multicolumn{2}{|c|}{ Constraints } \\
\hline Steering Angle $(\delta)$ & {$[-1.04-1.04] \mathrm{rad}$} \\
\hline Changing Rate of Steering Angle & {$[-0.26-0.26] \mathrm{rad}$} \\
\hline
\end{tabular}

\section{Simulation and results}

For the simulation process, the weights of the inputs and outputs of the controller were determined as it is shown in Table 2. It is worth to point out that non-zero values were assigned to the variables which have a reference to follow and the weight refers to the priority of the variables, where the high weight indicates high priority.

Table 2: Parameters' weights of MPC controller

\begin{tabular}{|c|c|}
\hline Parameter & Weight \\
\hline Change of steering angle & 0.1 \\
\hline Lateral deviation & 1 \\
\hline Yaw Angle & 0.1 \\
\hline Lateral Velocity & 0 \\
\hline Yaw Rate & 0 \\
\hline
\end{tabular}


After determining the weights of the parameters, the inputs of the MPC controller were defined (reference trajectory, longitudinal velocity, lateral deviation and relative yaw angle). The reference trajectory can be determined in two different ways, the first one is by using the Driving Scenario Designer Toolbox in MATLAB, where the waypoints can be determined in order to create lane -change maneuver, and in this case, the reference yaw angles and lateral positions will be determined automatically by the toolbox. The second way (used in this paper) is by generating future lane curvature, which is used to calculate the lateral deviation and relative yaw angle of the created lane (see Fig. 4). The longitudinal velocity is considered a constant parameter.

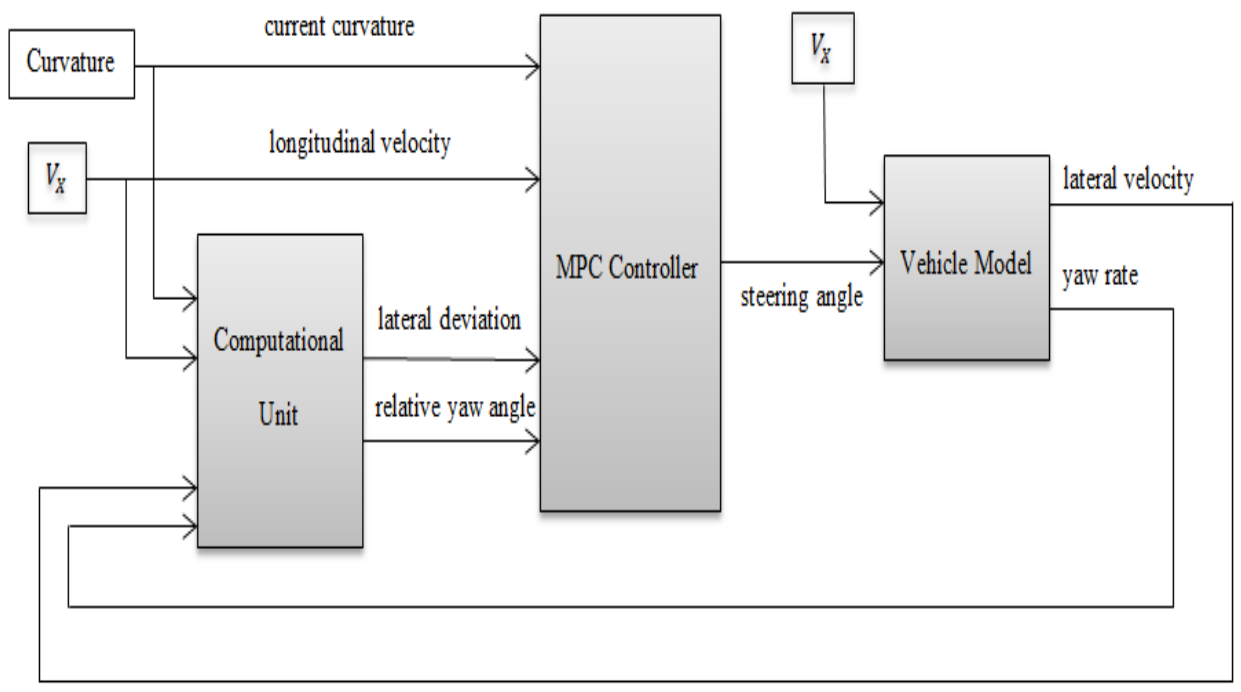

Figure 4: General Model of MPC and plant for Simulink

Fig. 5 shows the output control actions (steering angles) of the MPC controller after running the simulation, while Fig. 6 and Fig. 7 show the effects of these control actions on the lateral deviation and relative yaw angle which describe the performance of the controller. 


\section{Steering angle (deg)}

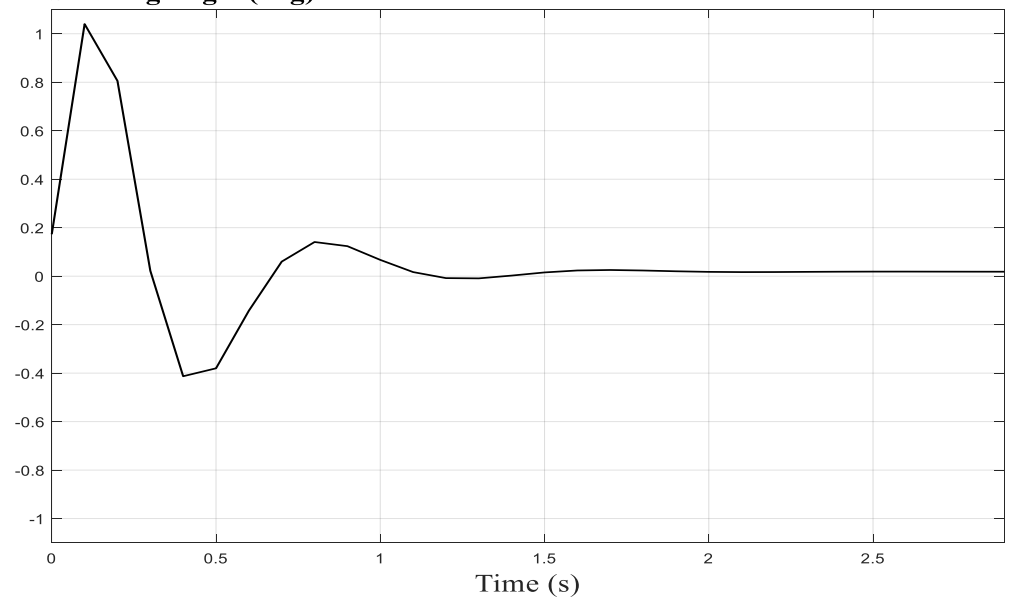

Figure 5: Control Actions of the MPC Controller

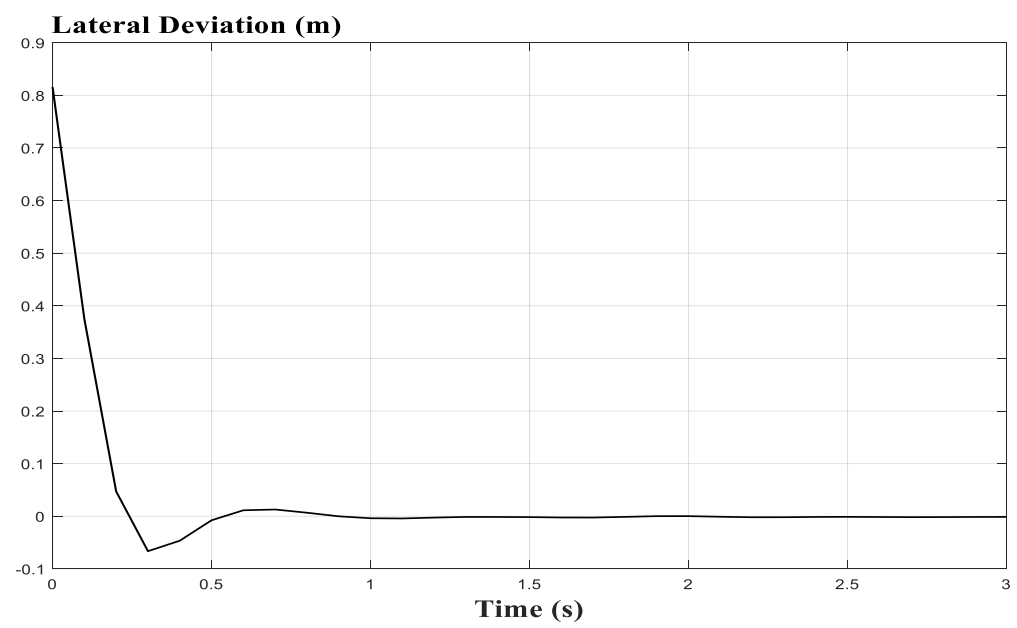

Figure 6: Lateral deviation resulting from the MPC implementation 


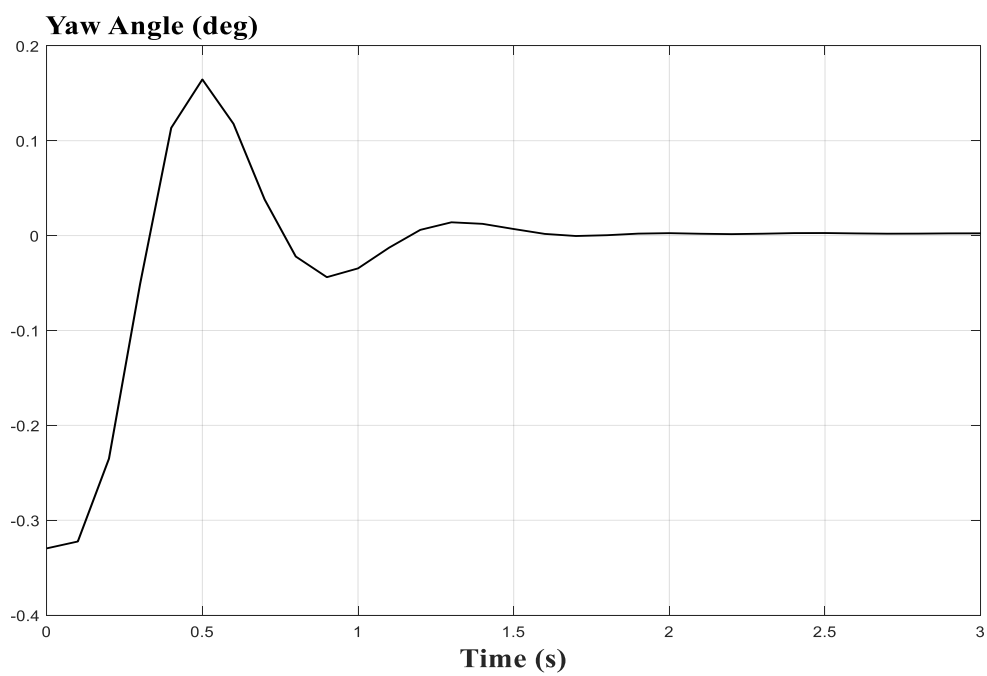

Figure 7: Relative yaw angle resulting from the MPC implementation

The obtained results show that the MPC controller succeeded in providing control actions that drive both relative yaw angle and lateral deviation to be very close to zero, meaning that, the designed MPC controller drove the autonomous vehicle along the reference path efficiently.

\section{Conclusions}

In this paper, a dynamic vehicle model and model based control strategy (model predictive controller) were used to implement trajectory tracking task for autonomous vehicle. The designed MPC controller succeeded in driving the vehicle within the desired trajectory by providing optimal control actions. Designing the vehicle model taking in consideration the internal and external forces and the well-designed MPC parameters played the essential role in achieving the satisfactory performance.

\section{Acknowledgements}

This research was supported by the European Union and the Hungarian State, co-financed by the European Regional Development Fund in the framework of GINOP-2.3.4-15-2016-00004 project, aimed to promote the cooperation between the higher education and the industry. 


\section{References}

[1] Fagnant, D. J., and Kockelman, K., "Preparing a nation for autonomous vehicles: opportunities, barriers and policy recommendations", Transportation Research Part A: Policy and Practice, pp. 167-181, 2015.

[2] Department for Transport, "Research on the Impacts of Connected and Autonomous Vehicles (CAVs) on Traffic Flow: Summary Report”, Atkins Ltd, 2017.

[3] Pendleton, S. D. et al., "Perception, planning, control, and coordination for autonomous vehicles", Machines, vol. 5, no. 1, pp. 1-54, 2017.

[4] Park, M., Lee, S., and Han, W., "Development of Steering Control System for Autonomous Vehicle Using Geometry-Based Path Tracking Algorithm", ETRI Journal, DOI: 10.4218/etrij.15.0114.0123, vol. 37, no. 3, pp. 617-625, 2015.

[5] Bacha, S., Ayad, M. Y., Saadi, R., Aboubou, A., Bahri, M., and Becherif, M., "Modeling and control technics for autonomous electric and hybrid vehicles path following", $5^{\text {th }}$ International Conference on Electric Engineering-Boumerdes-Boumerdes (ICEE-B), 2017.

[6] Katrakazas, C., Quddus, M., Chen,W.-H., and Deka, L., "Realtime motion planning methods for autonomous on-road driving: State-of-the-art and future research directions", Transp. Res. C: Emerg. Technol, vol. 60, pp. 416-422, 2015.

[7] Siegwart, R., Nourbakhsh, I., and Scaramuzza, D., "Introduction to Autonomous Mobile Robots", MIT Press, 2011.

[8] Aguiar, A. P., and Hespanha, J. P., "Hespanha Trajectory-tracking and path-following of underactuated autonomous vehicles with parametric modeling uncertainty", IEEE Transactions on Automatic Control, vol. 52, no. 8, pp. 1362-1379, 2007.

[9] Den Broeck, L. V., Diehl, M., Swevers, J., "Model predictive control approach for time optimal point-to-point motion control", Mechatronics, vol. 21, no. 7, pp.1203-1212, 2011.

[10] Amer N.H., Zamzuri H., Hudha K., and Kadir Z.A, "Modelling and control strategies in path tracking control for autonomous ground vehicles: A review of state of the art and challenges", J. Intell. Robot. Syst, DOI 10.1007/s10846-016-0442-0, vol. 86, no. 2, pp. 225254, 2017.

[11] Lucet, E., Lenain, R., and Grand, C., "Dynamic path tracking control of a vehicle on slippery terrain", Control Engineering Practice, DOI: 10.1016/j.conengprac.2015.05.008, vol. 42, pp. 60-73, 2015.

[12] Poussot-Vassal, C., Sename, O., Dugard, L., and Savaresi, S. M., "Vehicle dynamic stability improvements through gain-scheduled steering and braking control", Vehicle System Dynamics, vol. 49, no. 10, pp. 1597-1621, 2011.

[13] Benine-Neto, A., Scalzi, S., Mammar, S., and Netto, M., "Dynamic controller for lane keeping and obstacle avoidance assistance system", $13^{\text {th }}$ International IEEE Conference on Intelligent Transportation Systems (ITSC), pp. 1363-1368, 2010.

[14] Zhao, P., Chen, J., Mei, T. and Liang, H., "Dynamic Motion Planning for Autonomous Vehicle in Unknown Environments", in Proceedings of IEEE Intelligent Vehicles Symposium (IV), pp. 284-289, 2011.

[15] Reda, A., Bouzid, A., and Vásárhelyi, J., "Model Predictive Control for Automated Vehicle Steering", Acta Polytechnica Hungarica, vol. 17, no. 7, pp. 163-182, 2020.

[16] Turri, V., Carvalho, A., Tseng, H. E., Johansson, K. H., and Borrelli, F., "Linear model predictive control for lane keeping and obstacle avoidance on low curvature roads", Proc. IEEE Conf. Intell. Transp. Syst., pp. 378-383, 2013.

[17] Katriniok, A., Maschuw, J. P., Christen, F., Eckstein, L., and Abel, D., "Optimal vehicle dynamics control for combined longitudinal and lateral autonomous vehicle guidance", in Proc. IEEE Eur. Control Conf, pp. 974-979, 2013. 